\title{
Tailoring Electrostatic Attraction Interactions to Activate Persistent Room Temperature Phosphorescence from Doped Polyacrylonitrile Films
}

Hongzhuo Wu, Deliang Wang, Zheng Zhao, Dong Wang, Yu Xiong* and Ben Zhong Tang*

[a] Dr. H. Z. Wu, Dr. D. L Wang, Prof. Dr. D. Wang, Prof. Dr. Y. Xiong

Center for AIE Research, Shenzhen Key Laboratory of Polymer Science and Technology, Guangdong Research Center for Interfacial Engineering of Functional Materials, College of Materials Science and Engineering

College of Physics and Optoelectronic Engineering

Shenzhen University, Shenzhen 518060, China

E-mail: xiongyu@suz.edu.cn

[b] Prof. Dr. Y. Xiong, Prof. Dr. Z, Zhao, Prof. Dr. B. Z. Tang

HKUST Shenzhen Research Institute, Shenzhen 518057, China

[c] Prof. Dr. B. Z. Tang

Department of Chemistry and Hong Kong Branch of Chinese National Engineering Research Center for Tissue Restoration and Reconstruction, The Hong Kong University of Science and Technology Clear Water Bay, Kowloon, Hong Kong, China

E-mail: tangbenz@usk.hk

\begin{abstract}
Amorphous organic materials exhibiting room temperature phosphorescence (RTP) are good candidates for optoelectronic and biomedical applications. In this proof-of-concept work, we present a rational strategy to activate persistent RTP with a wide range of color from doped films in which electronrich organic phosphor as donor while electron-deficient polymer matrix as acceptor through electrostatic attraction interactions. By tailoring electrostatic attraction interactions between the donor and acceptor, an ultralong lifetime of $968.1 \mathrm{~ms}$ is achieved for doped film TBB-6OMe@PAN. Control experiments combined with theoretical calculations demonstrate that the electrostatic attraction interactions between organic phosphor and polymer matrix should be responsible for the persistent RTP of doped films. Besides, doped films show reversible thermal response and excellent stability in water, indicating an advantage of electrostatic attraction over hydrogen bond in terms of practical application.
\end{abstract}

Room temperature phosphorescence (RTP) materials show great superiority and prospect in applications of optoelectronics, anti-counterfeiting, sensing and bioimaging. ${ }^{[1-4]}$ At present, efficient RTP materials are limited to inorganic and organometallic compounds. These metal-containing compounds inevitably cause high-cost, environmental pollution and biotoxicity. To address these issues, metal-free organic RTP materials have received considerable attention because of inherent merits of low-cost, appreciable functionality, good biocompatibility and solution processability. However, it is extremely challenging to generate RTP of organic molecules due to inefficient intersystem crossing and ultrafast nonradiative decays.

In the past decade, a variety of organic RTP materials have been obtained by promoting spin-orbit coupling and suppressing nonradiative decays. ${ }^{[5-11]}$ Specifically, crystal engineering combined with $H$ aggregation, halogen bonding, $n, m^{*}$ transition, heavy atom effect and charge transfer have been demonstrated to be effective strategies to obtain efficient and persistent organic RTP materials. ${ }^{[12-17]}$ 
However, tricky growth condition and poor processability of organic crystals limited their practical applications. Thus, amorphous and flexible organic RTP materials are better candidates for future applications of bioimaging and foldable electronic devices. Nevertheless, it is difficult to suppress the nonradiative decays to allow efficient RTP emission in amorphous phase. Until recently, embedding organic phosphors into rigid matrices or macrocycle hosts have been proved to be efficient approaches to activate RTP from amorphous films. ${ }^{[9 a, 18-19]}$ For example, Adachi et al. reported an unique amorphous system showing ultralong RTP by doping deuterated organic phosphor into rigid steroidal compound. [19a] Kim et al. presented a synergetic strategy to obtain efficient RTP from doped polyvinyl alcohol (PVA) films by combining hydrogen bonding interactions with rigidification effect to minimize molecular motions. ${ }^{19 b]}$ Subsequently, polymer PVA became the most commonly used yet effective matrix to construct matrixassisted amorphous RTP systems. However, hydrogen bonds in doped PVA films are very sensitive to humidity, unfavorable for long-term stability in air. Besides, PVA matrix is only applicable to carboxylic acid, boric acid and hydroxyl or amine derivates with hydrogen bond donor or acceptor ability. In this context, it is essential to utilize a kind of non-covalent interaction with powerful environmental tolerance to trigger RTP from amorphous films.

It is well known that non-covalent interactions are involved in many biological processes. ${ }^{20-21]}$ Particularly, they play a key role in driving assembly processes for supramolecular systems. ${ }^{[22-23]}$ Noncovalent interactions mainly includes hydrogen and halogen bonding, electrostatic and hydrophobic interaction, $\pi$-effects and van der Waals forces. Attractive electrostatic interaction ${ }^{[24-25]}$ between the donor and acceptor appears universally if a donor and an acceptor with opposite electrostatic potential distributions are confined in a finite distance. ${ }^{[26]}$ Different from covalent bonds, electrostatic interaction has no directionality, which benefits effective contact and interaction between the donor and acceptor while binding them together. In addition, electrostatic interaction is not only stable at ambient condition, but also suitable for most organic molecules with polarity. Hence, tuning electrostatic attraction interaction to activate RTP from doped systems consist of a donor and an acceptor should be a rational and feasible strategy.

To prove this idea, a series of novel and tailor-made doped films were prepared by simply doping electron-rich organic phosphors into electron-deficient polymer matrix. As illustrated in Figure 1, polyacrylonitrile (PAN) is as glassy matrix but also as an acceptor due to abundant electron-withdrawing cyano groups. Aromatic compounds substituted with electron-donating methoxy groups are acted as donors. Active molecular motions can be restrained by binding organic phosphor and PAN matrix together through attractive electrostatic interactions, thus allowing organic phosphor emits long-lived RTP. Electrostatic attraction interactions between the donor and acceptor can be tuned by regulating molecular structure of organic phosphors, thus achieving ultralong and wide-color range RTP. Experimental results show that doped films consist of different organic phosphors and PAN matrix exhibit persistent RTP covering whole visible region. By tuning electrostatic attraction interactions between the donor and acceptor, an ultralong lifetime of $968.1 \mathrm{~ms}$ was achieved for doped film TBB-6OMe@PAN. Control experiments reveal that persistent RTP of doped films might be caused by electrostatic interactions between the donor and acceptor instead of rigidification effect. Theoretical studies confirm the formation of electrostatic attraction interactions between the donor and acceptor by mapping ESP distributions and calculating binding energy. The higher binding energy, the slower nonradiative decay rate, further proving that electrostatic attraction interactions between the donor and acceptor should be responsible for persistent RTP of doped films. The relationship between molecular structure and property reveals that organic phosphor with richer electrons is conducive to prolong lifetime by forming stronger electrostatic 
attraction interaction between the donor and acceptor. Besides, doped film TBB-6OMe@PAN still emit bright phosphorescence to naked eyes after soaking in water for over one month. This excellent waterresistance not only facilitates practical application, but also demonstrates an advantage of electrostatic attraction interaction over hydrogen bonding interaction.

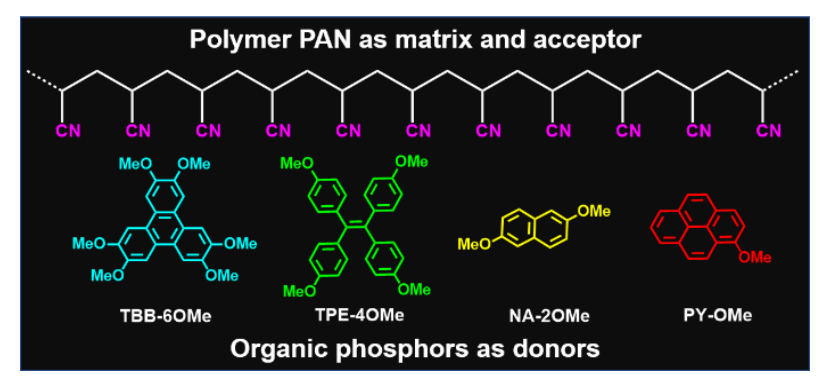

Figure 1. Chemical structures of polymer PAN and organic phosphors TBB-6OMe, TPE-4OMe, NA-2OMe and PYOMe.

As illustrated in Figure 1, doped films are composed of polymer PAN containing abundant cyano groups and organic phosphors substituted with methoxy groups, namely 2,3,6,7,10,11hexamethoxytribenzobenzene (TBB-6OMe), 1,1,2,2-tetrakis (4-methoxyphenyl) ethene (TPE-4OMe), 2,6-dimethoxynaphthalene (NA-2OMe) and methoxypyrene (PY-OMe), respectively. As polymer PAN and organic phosphors are soluble in N, N-dimethylformamide (DMF), doped films were prepared through drop-casting a DMF mixture of organic phosphor/PAN (1 mg/70 mg in $1 \mathrm{~mL}$ DMF) onto the quartz substrate with a thermal annealing treatment at $110{ }^{\circ} \mathrm{C}$ for $1 \mathrm{~h}$ to remove DMF residues. Thermal annealing treatment can also enhance rigidity of matrix and intermolecular interactions between organic phosphor and matrix to improve RTP property of doped films.
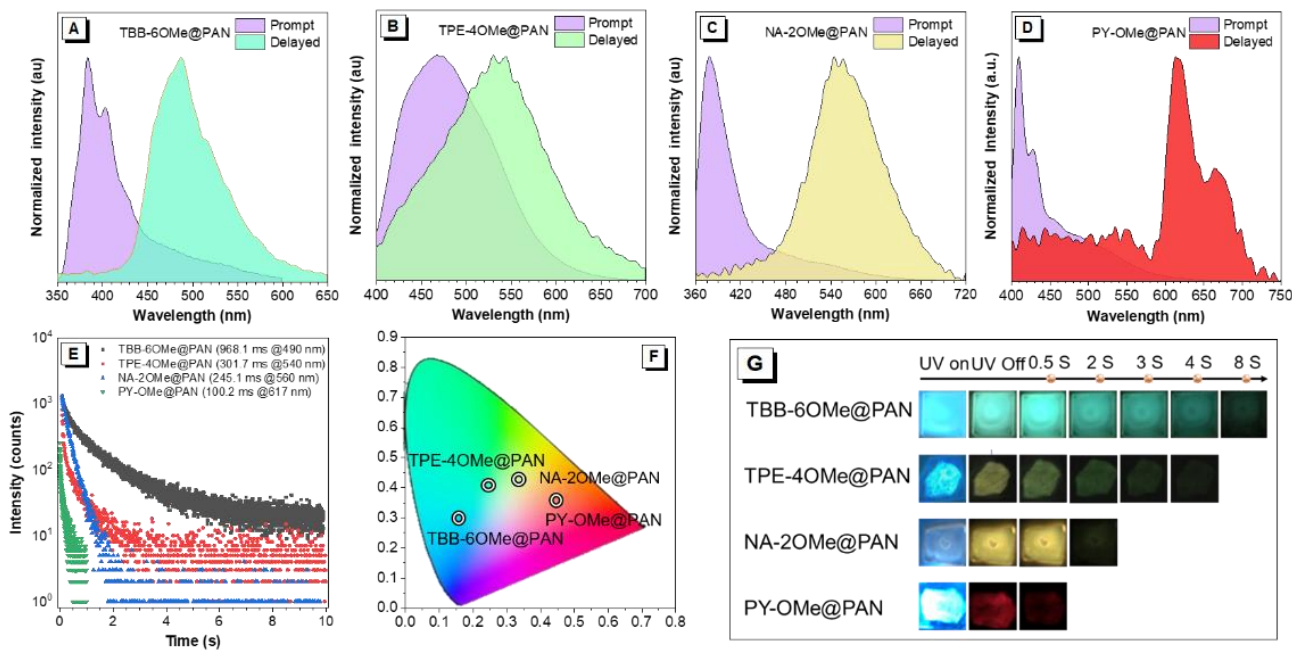

Figure 2. (A-D) Prompt and delayed PL spectra of doped films (delay $1 \mathrm{~ms}$ ); (E) phosphorescent decay curves of doped films; (F) CIE chromaticity diagram of delayed PL spectra of doped films; (G) Luminescence photographs of doped films under UV irradiation and after removal of UV irradiation with different duration time.

As expected, all the doped films exhibit obvious afterglow to naked eyes under ambient conditions. As shown in Figure 2G, after removing UV irradiation, blue, green, yellow and red afterglows were observed for doped film TBB-6OMe@PAN, TPE-4OMe@PAN, NA-2OMe@PAN and PY-OMe@PAN, respectively. Emission bands on the prompt PL spectra of doped films were ranged from 379 to $468 \mathrm{~nm}$, consistent 
with blue fluorescence observed under UV irradiation. After delaying $1 \mathrm{~ms}$, emission bands on the PL spectra were redshifted, revealing phosphorescence emissions derived from lower triplet states. In addition, lifetimes estimated by fitting the phosphorescent decay curves are in the scale of hundreds of milliseconds (Figure 2E), confirming the persistent RTP characteristics of doped films. It's worth mentioning that doped film TBB-6OMe@PAN exhibits an ultralong lifetime up to $968.1 \mathrm{~ms}$, which might be ascribed to strong electrostatic attraction interactions between the donor and acceptor. Besides, the Commission Internationale de l'Eclairage (CIE) coordinate of phosphorescence spectra moves gradually from blue to red region (Figure 2F), agreeing well with various afterglow colors observed to naked eyes. These experimental results demonstrate that long-lived RTP with various colors can be successfully achieved for doped films with a donor-acceptor construction in which electron-rich organic phosphor is as donor while electron-deficient polymer PAN is as acceptor.

As previous work reported, blue RTP with a short lifetime were detected from polymer PAN (Figure S3). ${ }^{[27]}$ To verify RTP attribution of doped films, phosphorescent emission and excitation spectra of doped films were compared with those of pure PAN film (Figure S4-6). Both emission and excitation bands of doped films are significantly different from pure PAN film, indicating that persistent RTP of doped films are not derived from the intrinsic triplet emission of polymer PAN. Then, phosphorescent emission spectra of doped films were compared with that of organic phosphors in both solution and solid state to determine whether RTP property of doped films is stemmed from intrinsic triplet emission of organic phosphors. As shown in Figure S7, the emission band of doped film TBB-6OMe@PAN and compound TBB-6OMe in dilute solution can overlap with each other, indicating that RTP feature of doped film TBB-6OMe@PAN origins from the single molecular phosphorescence of compound TBB-6OMe. However, the emission bands of powders NA-2OMe and PY-OMe are overlapped well with that of doped films NA-2OMe@PAN and PY-OMe@PAN, respectively, which implies that persistent RTP of doped films NA-2OMe@PAN and PY-OMe@PAN can be ascribed to the aggregate phosphorescence of compounds NA-2OMe and PYOMe. Unlike other doped films, the emission band of doped film TPE-4OMe@PAN falls between the dilute solution and solid state's emission bands of compound TPE-4OMe. In addition, absorption and phosphorescent emission spectra of doped film TPE-4OMe@PAN display no obvious shift with increasing doping concentration of compound TPE-4OMe (Figure S9-10). Thus, the RTP property of doped film TPE4OMe@PAN should be derived from the single molecular phosphorescence of compound TPE-4OMe.

Single crystal X-ray diffraction (XRD) analysis were performed onto organic phosphors to gain insight into the impact of molecular geometry and intermolecular interactions on the attribution of RTP of doped films (Figure S11-12). Fused-ring compounds TBB-6OMe, NA-2OMe and PY-OMe have good planarity, whereas compound TPE-4OMe exhibits a non-planar conformation (Figure S11). № $\pi \cdots \pi$ stacking is observed in crystal TBB-6OMe with a large conjugated plane, which might be due to electron repulsion between electron-rich cores. Without $\pi \cdots \pi$ stacking interaction, compound TBB-6OMe tends to form a monodisperse state in doped film. On the contrary, strong $\pi \cdots \pi$ stacking interactions exist in crystal PYOMe, which is conducive to form aggregate state when doping compound PY-OMe into PAN matrix. Crystal NA-2OMe displays a tight packing style with strong intermolecular interactions, which also facilitates to form aggregate state when doping compound NA-2OMe into PAN matrix. Notably, the highly distorted geometry and weak intermolecular interactions were observed in crystal TPE-4OMe, which hinders the aggregate formation when doping compound TPE-4OMe into PAN matrix. Single crystal XRD data demonstrate that RTP attribution of doped films are determined by the single molecular geometry and intermolecular interactions of organic phosphors. 


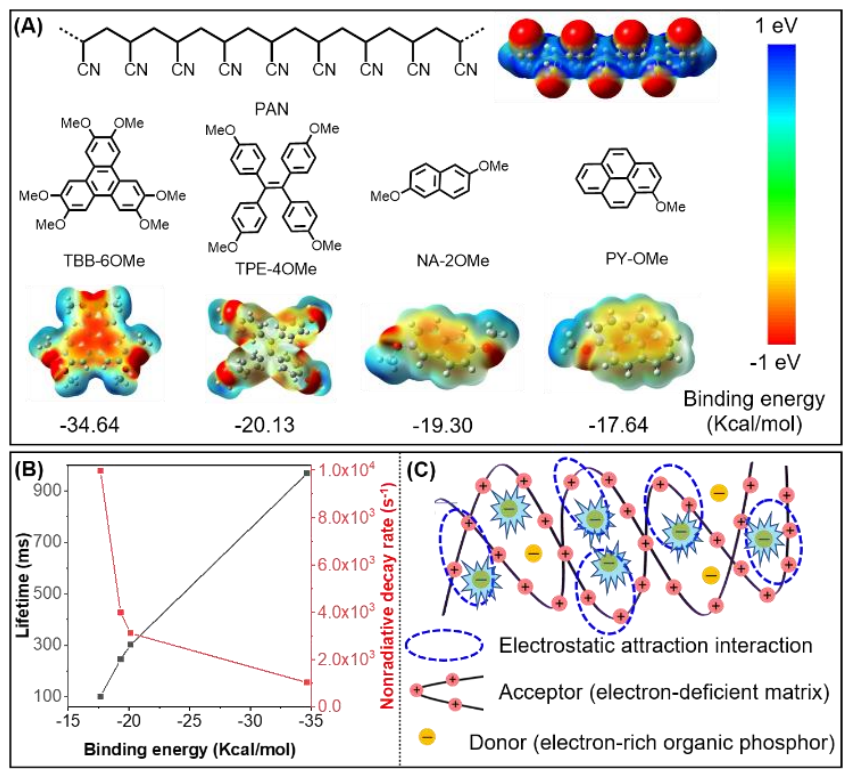

Figure 3. (A) Molecular structures and calculated ESP distribution of polymer PAN and organic phosphors, binding energy between organic phosphors and PAN; (B) Relationships between binding energy, lifetime and nonradiative decay rate; (C) illustration diagram of RTP emission mechanism.

To further understand the underlying mechanism of RTP emission, we first carried out control experiments. Polymers polymethyl methacrylate (PMMA) and polystyrene (PS) which are not electrondeficient acceptors but have a similar recorded $\mathrm{Tg}$ value $\left(\sim 105\right.$ and $\left.95^{\circ} \mathrm{C}\right)$ to polymer $\mathrm{PAN}\left(\sim 95^{\circ} \mathrm{C}\right)$ were selected as a reference to assess rigidification effect of matrix. Surprisingly, doped PMMA and PS films exhibit very weak or even no detectable phosphoresce under ambient conditions, indicating that rigidification effect alone is not sufficient to trigger RTP of organic phosphors in doped films. Compared to PMMA or PS, PAN is not only as matrix, but also as acceptor due to abundant cyano groups with strong electron-withdrawing ability. If electron-rich organic phosphors are doped into PAN matrix, electrostatic attraction interactions should be formed between the donor and acceptor. To confirm this hypothesis, the electrostatic potential (ESP) distribution on the molecular surface of PAN and organic phosphors TBB6OMe, TPE-4OMe, NA-2OMe and PY-OMe were mapped by DFT calculations to qualitatively describe the electrostatic interactions between the donor and acceptor. As illustrated in Figure 3A, the ESP value on the main chain surface of PAN is positive, whereas that on the core surface of organic phosphor is negative. As a result, the negative core of organic phosphor will attract positive main chain of PAN to combine them together, thus leading to restricted molecular motions. The binding energies between organic phosphors and polymer PAN were calculated to be $37.40,22.08,21.21$ and $19.35 \mathrm{Kcal} / \mathrm{mol}$ for doped films TBB-6OMe@PAN, TPE-4OMe@PAN, NA-2OMe@PAN and PY-OMe@PAN, respectively, implying the strong electrostatic attraction interactions between the donor and acceptor. As shown in Figure 3B, with binding energy increased, the nonradiative decay rate is decreased while the lifetime is increased, which demonstrates that suppressing nonradiative decays by strong electrostatic attraction interactions between organic phosphor and PAN matrix should be responsible for the persistent RTP of doped films. The proposed mechanism is illustrated in Figure $3 \mathrm{C}$, electron-deficient polymer PAN is as acceptor and electron-rich organic phosphors are as donors. When doping organic phosphors into polymer PAN, the electrostatic attraction interactions will present between them and bind them together to restrain molecular motions, thus activating persistent RTP of doped films. Besides, due to the richest 
electrons of TBB-6OMe, the binding energy between organic phosphor TBB-6OMe and PAN matrix is the highest, thus leading to an ultralong lifetime of doped film TBB-6OMe@PAN.

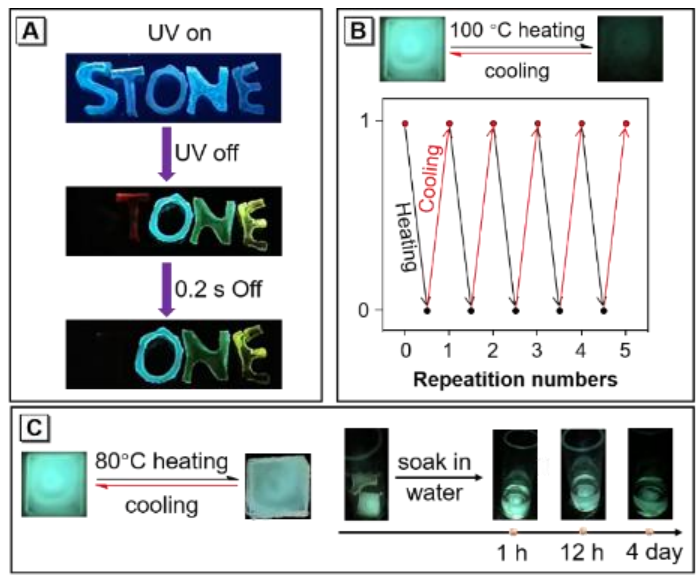

Figure 4. (A) With UV light on, the word "STONE" consists of doped films TPE@PAN, PY-OMe@PAN, TBB6OMe@PAN, TPE-4OMe and NA-2OMe@PAN in turn is visible to naked eyes. After removing UV light, the word "STONE" is turned into the new word "TONE" showing various colors. With UV light off for $0.2 \mathrm{~s}$, the word "TONE" is turned into another new word "ONE"; (B) illustration of reversible temperature response of doped film TBB6OMe@PAN; (C) illustration of thermal and water resistance of doped film TBB-6OMe@PAN.

After revealing the RTP emission mechanism, we finally studied the potential applications of doped films. Different afterglow colour and emission lifetime of these doped films makes them promising for advanced-encryption by utilizing time-gated technology. As shown in Figure 4A, the word "STONE" is composed of doped films TPE@PAN, PY-OMe@PAN, TBB-6OMe@PAN, TPE-4OMe and NA2OMe@PAN in turn. Under UV light excitation, the word "STONE" emits blue fluorescence. After turning off the UV light, the letter "S" written with doped film TPE@PAN is disappeared because of short-lived fluorescence. Thus, the word "STONE" turns into a new word "TONE" with each letter emitting varied afterglow colours. When the UV light is removed for $0.2 \mathrm{~s}$, the letter "T" written with doped film PYOMe@PAN showing short-lived RTP is disappeared too. As a result, the word "TONE" turns into another new word "ONE". Furthermore, thermal and moisture stabilities of doped films were tested. As show in Figure 4B, doped film TBB-6OMe@PAN displays bright green afterglow lasting for several seconds to naked eyes. When heating to $100^{\circ} \mathrm{C}$, the green afterglow is completely quenched but recovered well after cooling to room temperature. By repeating the heating and cooling cycles, the on-off recording via reversible temperature response of persistent RTP signal is realized. Besides, when heating to $80^{\circ} \mathrm{C}$, below the Tg of PAN matrix, the green afterglow becomes weaker but still maintain bright to naked eyes. To our surprise, persistent RTP of doped film TBB-6OMe@PAN remain bright after soaking in water for several days and still visible to naked eyes after soaking for over one month, manifesting fairly good water resistance. As we all known, doped PVA films depending on hydrogen bonding interaction to generate RTP are very sensitive to humidity, which is detrimental to practical applications. Therefore, the excellent water resistance of doped PAN films is not only favourable for future applications, but also demonstrating an advantage of electrostatic attraction interaction over hydrogen bonding interaction.

In summary, persistent RTP was successfully achieved from amorphous films by doping electron-rich organic phosphors into electron-deficient polymer PAN. By tuning molecular structure of organic phosphors, an ultralong lifetime of 968.1 ms was obtained for doped film TBB-6OMe@PAN. Spectroscopic data demonstrate that persistent RTP of doped films origins from the intrinsic triplet 
emission of organic phosphors in single molecular or aggregate state. Single crystal XRD data reveal that RTP attribution of doped films mainly depend on the molecular geometry and intermolecular interactions of organic phosphors. A combination of control experiments and theoretical calculations prove that electrostatic attraction interactions between electron-rich organic phosphor and electron-deficient PAN matrix instead of rigidification effect should be responsible for the persistent RTP of doped films. Besides, doped film TBB-6OMe@PAN exhibits reversible thermal response and excellent water resistance. This instructive design strategy to activate persistent RTP from amorphous films by electrostatic attraction interactions between the donor and acceptor opens up a new direction for the exploration of organic RTP materials.

\section{Acknowledgements}

This work was financially supported by the National Natural Science Foundation of China $(21805233,21788102)$, the Science and Technology Plan of Shenzhen (JCYJ20180306174910791, JCYJ20160509170535223), the Research Grants Council of Hong Kong (16305618, 16305518, N_HKUST609/19, and C6009-17G), and the Innovation and Technology Commission (ITC-CNERC14SC01).

\section{References}

[1] a) R. Kabe, N. Notsuka, K. Yoshida, C. Adachi, Adv. Mater. 2016, 28, 655-660. b) T. Wang, X. Su, X. Zhang, X. Nie, L. Huang, X. Zhang, X. Sun, Y. Luo, G. Zhang, Adv. Mater. 2019, 31, 1904273.

[2] a) L. Gu, H. Shi, L. Bian, M. Gu, K. Ling, X. Wang, H. Ma, S. Cai, W. Ning, L. Fu, H. Wang, S. Wang, Y. Gao, W. Yao, F. Huo, Y. Tao, Z. An, X. Liu, W. Huang, Nat. Photonics 2019, 13, 406-411; b) Y. Su, S. Z. F. Phua, Y. Li, X. Zhou, D. Jana, G. Liu, W. Q. Lim, W. K. Ong, C. Yang, Y. Zhao, Sci. Adv. 2018, 4, eaas9732; c) P. Long, Y. Feng, Chen Cao, Y. Li, J. Han, S. Li, C. Peng, Z. Li, W. Feng, Adv. Funct. Mater. 2018, 28, 1800791.

[3] a) A. Fermi, G. Bergamini, M. Roy, M. Gingras, P. Ceroni, J. Am. Chem. Soc. 2014, 136, 6395-6400; b) C. A. DeRosa, C. Kerr, Z. Y. Fan, M. Kolpaczynska, A. S. Mathew, R. E. Evans, G. Q. Zhang, C. L. Fraser, ACS Appl. Mater. Interfaces 2015, 7, 23633-23643; c) L. Huang, B. Chen, X. Zhang, C. O. Trindle, F. Liao, Y. Wang, H. Miao, Y. Luo, G. Zhang, Angew. Chem. Int. Ed. 2018, 57, 16046-16050.

[4] a) W. Zhou, Y. Chen, Q. Yu, H. Zhang, Z. Liu, X. Dai, J. Li, Y. Liu, Nat. Commun.2020, 11, 4655; b) G. Zhang, G. M. Palmer, M. W. Dewhirst, C. L. Fraser, Nat. Mater. 2009, 8, 747-751; c) X. Zhen, Y. Tao, Z. An, P. Chen, C. Xu, R. Chen, W. Huang, K. Pu, Adv. Mater. 2017, 29, 1606665.

[5] M. Baroncini, G. Bergamini, P. Ceroni, Chem. Commun. 2017, 53, 2081-2093.

[6] Kenry, C. Chen, B. Liu, Nat. Commun.2017, 10, 2111.

[7] W. Zhao, Z. He, B. Tang, Nat Rev Mater. 2020, 5, 869.

[8] T. Zhang, X. Ma, H. Wu, L. Zhu, Y. Zhao, H. Tian, Angew. Chem. Int. Ed. 2020, 59, 11206-11216.

[9] a) N. Gan, H. Shi, Z. An, W. Huang, Adv. Funct. Mater. 2018, 28, 1802657; b) S. Xu, R. Chen, C. Zheng, W. Huang, Adv. Mater. 2016, 28, 9920-9940.

[10] S. Hirata, Adv. Optical Mater. 2017, 5, 1700116.

[11] J. Yang, M. Fang, Z. Li, InfoMat. 2020, 2, 791-806.

[12] W. Yuan, X. Shen, H. Zhao, Jacky W. Y. Lam, L. Tang, P. Lu, C. Wang, Y. Liu, Z. Wang, Q. Zheng, J. Sun, Y. Ma, B. Tang, J. Phys. Chem. C. 2010, 114, 6090-6099.

[13] Z. An, C. Zheng, Y. Tao, R. Chen, H. Shi, T. Chen, Z. Wang, H. Li, R. Deng, X. Liu, W. Huang, Nat. Mater. 2015, 14, 685-690.

[14] a) O. Bolton, K. Lee, H. J. Kim, K. Y. Lin, J. Kim, Nat. Chem. 2011, 3, 205-210; b) H. Gao, X. Zhao, H. Wang, X. 
Pang, W. Jin, Cryst. Growth Des. 2012, 12, 4377-4387; c) L. Xiao, Y. Wu, Z. Yu, Z. Xu, J. Li, Y. Liu, J. Yao, H. Fu, Chem. Eur. J. 2018, 24, 1801-1805

[15] a) W. Zhao, Z. He, J. W. Y. Lam, Q. Peng, H. Ma, Z. Shuai, G. Bai, J. Hao, B. Z. Tang, Chem 2016, 1, 592-602; b) H. Ma, Q. Peng, Z. An, W. Huang, Z. Shuai, J. Am. Chem. Soc. 2019, 141, 1010-1015.

[16] a) D. R. Lee, K. H. Lee, W. Shao, C. L. Kim, J. Kim, J. Y. Lee, Chem. Mater. 2020, 32, 2583-2592; b) K. Kanosue, S. Ando, ACS Macro Lett. 2016, 5, 1301-1305.

[17] a) X. Chen, C. Xu, T. Wang, C. Zhou, J. Du, Z. Wang, H. Xu, T. Xie, G. Bi, J. Jiang. X. Zhang, J. N. Demas, C. O. Trindle, Y. Luo, G. Zhang, Angew. Chem. Int. Ed. 2016, 55, 9872-9876; b) C. Chen , A. S. Batsanov, P. Pander,YT Hsu, Z. Chi, F. B. Dias, M. R. Bryce, Angew. Chem. Int. Ed. 2018, 57, 16407-16411; c) I. Bhattacharjee, N. Acharya, S. Karmakar, D. Ray, J. Phys. Chem. C. 2018, 122, 21589-21597.

[18] a) X. Ma, J. Wang, H. Tian, Acc. Chem. Res. 2019, 52, 738-748; b) M. Fang, J. Yang, Z. Li, Chinese J. Polym. Sci. 2019, 37, 383-393.

[19] a) S. Hirata , K. Totani , J. Zhang , T. Yamashita , H. Kaji, S. R. Marder, T. Watanabe, C. Adachi, Adv. Funct. Mater. 2013, 23, 3386-3397; b) M. S. Kwon, D. Lee, S. Seo, J.Jung, J. Kim, Angew. Chem. Int. Ed. 2014, 53, 11177-11181; c) Y. Su, Y. Zhang, Z. Wang, W. Gao, P. Jia, D. Zhang, C. Yang, Y. Li, Y. Zhao, Angew. Chem. Int. Ed. 2020, 59, 9967-9971; d) R. Gao, X. Fang, D. Yan, J. Mater. Chem. C, 2018, 6, 4444-4449; e) S. Kuila, S. Garain, S. Bandi, S. J. George, Adv. Funct. Mater. 2020, 2003693; f) Z. Zhang, W. Xu, W. Xu, J. Niu, X. Sun, Y. Liu, Angew. Chem. Int. Ed. 2020, 59, 18748-18754; g) J. Wang, Z. Huang, X. Ma, H. Tian, Angew. Chem. Int. Ed. 2020, 59, 9928-9933.

[20] D. J. McClements, Biotechnol. Adv. 2006, 24, 621-625.

[21] J. Cerny, P. Hobza, Phys. Chem. Chem. Phys. 2007, 9, 5291-5303.

[22] K. T. Mahmudov, M. N. Kopylovich, M. F. C. Guedes da Silva, A. J. L. Pombeiro, Coord. Chem. Rev. 2017, 345, 54-72.

[23] C. E. Boott, A. Nazemi, I. Manners, Angew. Chem. Int. Ed. 2015, 54, 13876-13894

[24] R. S. J. Proctor, A. C. Colgan, R. J. Phipps, Nat. Chem. 2020.

[25] H.-X. Zhou, X. Pang, Chem. Rev. 2018, 118, 1691-1741.

[26] a) M. A. Spackman, J. J. McKinnon, D. Jayatilaka, CrystEngComm. 2008, 10, 377-388; b) Y. Xu, H. Yao, L. Ma, L. Hong, J. Li, Q. Liao, Y. Zu, J. Wang, M. Gao, L. Ye, J. Hou, Angew. Chem. Int. Ed. 2020, 59, 9004-9010.

[27] Q. Zhou, B. Cao, C. Zhu, S. Xu, Y. Gong, W. Yuan, Y. Zhang, Small. 2016, 12, 6586-6592. 